\title{
Reward Acts on the pFC to Enhance Distractor Resistance of Working Memory Representations
}

\author{
Sean James Fallon ${ }^{1}$ and Roshan Cools ${ }^{1,2}$
}

\begin{abstract}
Working memory and reward processing are often thought to be separate, unrelated processes. However, most daily activities involve integrating these two types of information, and the two processes rarely, if ever, occur in isolation. Here, we show that working memory and reward interact in a task-dependent manner and that this task-dependent interaction involves modulation of the $\mathrm{pFC}$ by the ventral striatum. Specifically, BOLD signal during gains relative to losses in the ventral striatum and $\mathrm{pFC}$ was asso-
\end{abstract}

\section{INTRODUCTION}

Efficient working memory and reward processing are both essential functions in everyday life. Most daily tasks involve either actively integrating or segregating these two types of information. For example, during decisionmaking, we need to maintain information and integrate it with reward-related information. Conversely, we also need to be able to perform cognitive tasks without letting reward-related information impinge upon our performance. For example, good or bad news should not distract us from performing our current task.

Frontostriatal circuitry has been implicated in both reward processing and working memory (e.g., Aarts, Holstein, \& Cools, 2011; van Schouwenburg, Aarts, \& Cools, 2010; Rowe et al., 2008; Hazy, Frank, \& O’Reilly, 2007; Lawrence, Sahakian, \& Robbins, 1998). Specifically, reward processing is well known to implicate the ventral striatum (Delgado, Nystrom, Fissell, Noll, \& Fiez, 2000), whereas working memory has been shown to involve, among other regions, the lateral pFC (Petrides, 1996; Chao \& Knight, 1995; Funahashi, Bruce, \& GoldmanRakic, 1993; Owen, Downes, Sahakian, Polkey, \& Robbins, 1990) and the dorsal striatum (Baier et al., 2010; Collins, Wilkinson, Everitt, Robbins, \& Roberts, 2000). Despite this apparent segregation, the anatomical organization of frontostriatal circuitry affords several opportunities for interaction between reward and working memory. These points of interaction range from direct gating and/or stabilization of prefrontal working memory represen-

\footnotetext{
${ }^{1}$ Radboud University Nijmegen, ${ }^{2}$ Radboud University Medical Centre Nijmegen
}

ciated not only with enhanced distractor resistance but also with impairment in the ability to update working memory representations. Furthermore, the effect of reward on working memory was accompanied by differential coupling between the ventral striatum and ignore-related regions in the pFC. Together, these data demonstrate that reward-related signals modulate the balance between cognitive stability and cognitive flexibility by altering functional coupling between the ventral striatum and the pFC. tations by midbrain reward signals (Braver \& Cohen, 2000) to indirect modulation of prefrontal function by reward via striatal go and no-go pathways (Moustafa, Sherman, \& Frank, 2008; Hazy et al., 2007; Frank, 2005). However, relatively few studies (Krawczyk \& D’Esposito, 2013; Marquand et al., 2011; Kennerley \& Wallis, 2009a, 2009b; Krawczyk, Gazzaley, \& D’Esposito, 2007) have investigated the (neural) interaction between reward and working memory. Here, we examine this interaction and its underlying frontostriatal mechanisms using fMRI in young healthy volunteers.

Everyday experience might suggest that people perform better on a cognitive task in the context of reward. Thus, ostensibly, the relationship between reward and working memory is simple: Reward enhances cognitive performance. This intuition is supported by human and animal work suggesting that reward enhances goaldirected behavior through increasing dopamine levels (Wise, 2004; Robbins \& Everitt, 1996; Dickinson \& Balleine, 1994). However, existing knowledge about the dopaminergic basis of reward and working memory points to a more complicated interaction. Specifically, recent evidence suggests that dopamine has opposing effects on distinct subcomponent processes of working memory. Thus, reward, which is commonly associated with dopamine release (Tobler, Fiorillo, \& Schultz, 2005; Schultz, Dayan, \& Montague, 1997), may affect working memory in a task-selective manner (Cools \& D’Esposito, 2011; Durstewitz \& Seamans, 2008). We put forward two hypotheses.

The first hypothesis is based on early insights that the dopamine increase triggered by reward might act directly in the pFC to modulate working memory by enhancing 
the signal-to-noise ratio of task-relevant representations (Braver \& Barch, 2002; Servan-Schreiber, Printz, \& Cohen, 1990). These ideas have been worked out in biophysically detailed computational models that have suggested that dopamine levels in the pFC might have opposite effects on the ability to ignore (cognitive stability) and update (cognitive flexibility) items in working memory (Durstewitz \& Seamans, 2008). According to this modeling work, dopamine alters the degree to which working memory representations are susceptible to interference (distraction). This change in distractor resistance, however, is accompanied by a change in the degree to which working memory representations can be updated, thus leading to altered flexibility. Furthermore, the sparsity of dopamine reuptake transporters in the $\mathrm{pFC}$ implies that these phasic dopamine impulses can influence neuronal processing over an extended period of time (Seamans \& Yang, 2004). Therefore, within the above framework, the increase in dopamine that accompanies unexpected reward (and the decrease in dopamine that accompanies unexpected punishment) might alter the balance between distractor resistance and flexible updating of items in working memory. These reward effects should be accompanied by altered signaling in the pFC during distractor resistance versus updating and/or altered connectivity between reward-related regions such as the ventral striatum and the pFC.

An alternative hypothesis is grounded in more recent neurocomputational models that have highlighted a common role for dopamine in the striatum in reinforcement learning and in working memory (Maia \& Frank, 2011; Frank, 2005). According to this work, a division of labor exists between the direct (go) pathway and the indirect (no-go) pathway of the BG, with the former allowing and the latter inhibiting items from entering working memory (Hazy et al., 2007). Importantly, dopamine is thought to increase go but decrease no-go activity (Gerfen \& Surmeier, 2011), thus influencing whether items are gated into versus filtered out from working memory. Therefore, within this framework, reward might modulate the balance between updating and filtering out new information from working memory by acting on the striatum. According to this hypothesis reward effects on working memory might be accompanied foremost by altered signaling in the striatum during updating versus distractor resistance.

To test these hypotheses, we modified a classic delayed match-to-sample test of working memory by including two additional phases in the delay between encoding and probe. First, participants were presented with an unexpected financial gain or loss. After this, participants were presented with intervening stimuli, that is, new items that either had to be ignored or updated into working memory. This allowed us to selectively examine the effects of unexpected gains and losses on subsequent updating and ignoring of information without altering how this initial information was encoded.

\section{METHODS}

\section{Participants}

Twenty-four healthy participants (11 men) were recruited to take part in the fMRI study. Their mean age was 22 years (range $=18-30)$. Data from four participants were excluded from the analysis; two were excluded because of computer failure, one was excluded because of technical problems with the fMRI scanner, and one withdrew from the experiment after $10 \mathrm{~min}$ in the scanner. The study conformed to the Helsinki Declaration of 1975 and was approved by the local ethics committee (Commissie Mensgebonden Onderzoek Arnhem-Nijmegen number 2001/095). The exclusion criteria for participation in the study were as follows: uncorrected visual impairment (e.g., color blindness), history of neurological or psychiatric disorder, history of medical treatment to head or neck, history of asthma, history of disorder that may affect metabolism or circulation (diabetes, hypertension, cardiac arrhythmia), currently taking over-the-counter medications, pregnancy, metallic implants, current smoker, consumption of $>20$ units alcohol per week, history of hard drug use (e.g., heroin or cocaine), nonnative Dutch speaking, left-handedness, tattoos, nonremovable body piercings, claustrophobia, and frequent gambling (e.g., casino, slot machines).

\section{Design and Procedure}

Before entering the scanner, participants received standardized instructions, completed two practice blocks, and confirmed to the experimenter that they fully understood the task. The task was designed to probe the extent to which the experience of an unexpected gain or unexpected loss (during the delay period) modulated working memory performance and how this modulation was modified according to task demands (ignoring vs. updating information). To examine this, we modified the standard delayed match-to-sample task to include two extra components during the delay period. In a standard delayed match-to-sample design, participants have to retain mnemonic material (the targets) over a delay period until a probe phase in which participants have to decide whether the probe matches (one of) the target(s). The two extra components included in this design were (i) the presentation of either an unexpected financial gain, loss, or neutral outcome after initial encoding (upon completion of a gamble task) and (ii) the presentation of new stimuli that had to be either ignored (ignore condition) or encoded as targets, thus displacing the original mnemonic information (update condition). We also included control trials in which no intervening stimuli were presented (no-interference condition). Thus, we employed a 3 (gain/loss/neutral) $\times 3$ (ignore/ update/no-interference) factorial design (Figure 1).

The task was programmed using the Matlab psychophysics toolbox version 3 (Kleiner, Brainard, \& Pelli, 


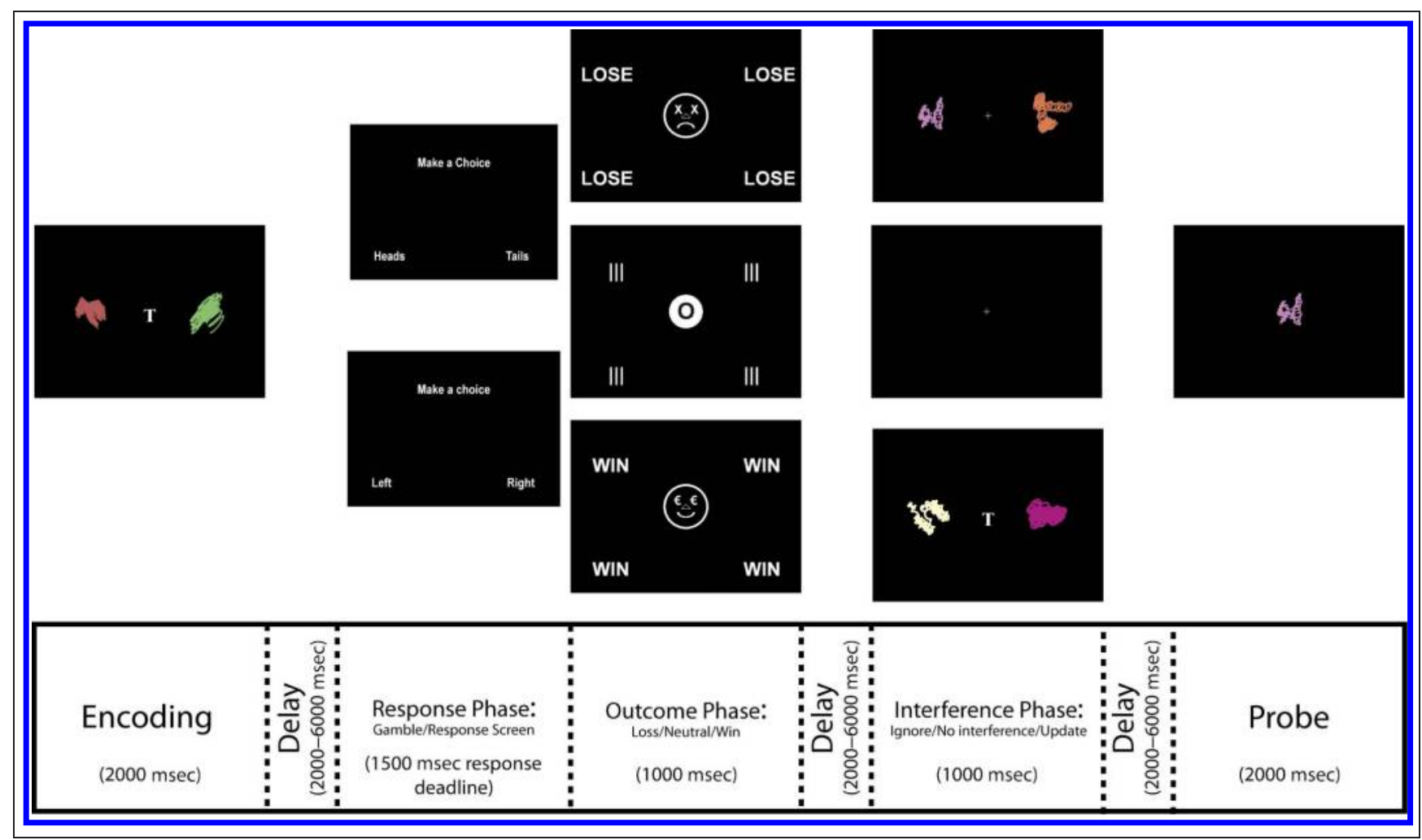

Figure 1. An illustration of the task. Participants were presented with two stimuli that had to be encoded (2000 msec). Then, after a variable delay period (2000-6000 msec), they were presented with a response screen that led to the presentation of a gain, neutral, or loss outcome. Then, after another variable delay period (2000-6000 msec), participants were presented with novel stimuli (1000 msec) that had to be ignored or used for working memory updating (displacing the original targets). There was also a no-interference condition in which only a fixation cross appeared $(1000 \mathrm{msec})$. Finally, after another variable delay period $(2000-6000 \mathrm{msec})$, participants were presented with a probe item, in which they had a maximum of 2000 msec to respond.

2007; Brainard, 1997). Participants completed 180 trials, distributed across five sessions. Ignore and update trials were blocked to reduce task-switching effects. Each of the five sessions contained four blocks (two update and two ignore blocks). Each block contained nine trials: six update or ignore trials and three no-interference trials. The no-interference trials ensured that participants could not anticipate whether the initial encoding stimuli had to be remembered. During each trial, participants could gain or lose (or receive no) money during the delay period (see below for more details). Within each block, each outcome was delivered with equal frequency, that is, for the nine trials within a block, there were three gain, neutral, and loss outcomes.

Each trial consisted of four distinct phases separated by three delay periods (Figure 1). Delay periods consisted of a blank screen and were randomly jittered to last between 2000 and 6000 msec to improve the efficiency of hemodynamic response estimation.

\section{Encoding Phase}

In this phase, participants were presented with the letter " $T$ " in the middle of the screen flanked by two computergenerated "spirographs," which were composed of differ- ent RGB elements. These stimuli remained on the screen for 2000 msec. Participants were instructed that the presence of a " $T$ " in the center of the screen indicated that the stimuli had to be remembered as the targets and that those stimuli remained the target stimuli until they saw a new set of target stimuli.

\section{Outcome Phase}

On the gain and loss trials, participants were presented with a screen inviting them to guess the outcome of a coin toss (Figure 1). They could make a head or tails response with their index or middle finger, respectively. After this, participants were presented with two possible outcomes: a "LOSS" screen, which featured a sad face flanked by the word "Lose" presented at the four corners of the screen (Figure 1) and the presentation of a negative auditory tone ("horn"), or a "WIN" screen, which featured a happy face flanked by four presentations of the word "Win" in each corner of the screen accompanied by a positive auditory tone (cash register). Participants were told that a "LOSS" screen indicated that they had lost $€ 1$ and a "WIN" screen indicated they had won $€ 1$. It might be noted here that gains and losses were not dependent on performance and equally frequent. 
Accordingly, there was no positive net gain or loss. In a control condition, participants were not presented with a gamble screen but were simply asked to make a left or right response, after which they saw a neutral screen, which featured a neutral face with "|||" presented at the four corners of the screen. Each of the three outcomes appeared on the screen for $1500 \mathrm{msec}$.

\section{Interference Phase}

There were two experimental conditions: ignore and update. In the ignore condition, participants were shown a new set of stimuli that they had to ignore. Conversely, in the update condition, participants were shown a new set of stimuli that displaced the previous set of stimuli as the target stimuli. There was also a control condition in which only a fixation cross appeared on the screen (nointerference).

\section{Probe Phase}

In this phase, participants were presented with a single stimulus in the center of the screen. Participants had to respond according to whether this probe was a target or nontarget stimulus with their middle or index finger, respectively. There was an equal probability of the probe being a target or nontarget stimulus. Half of nontarget stimuli were novel stimuli; the other half were stimuli that featured, in the case of ignore blocks, as distractors or, in the case of update blocks, as stimuli that were initially encoded as target stimuli but which were displaced by targets in the intervening period.

Each trial was separated by an intertribal interval of $2000 \mathrm{msec}$, and at the end of each block, participants were presented with a screen that informed them of their accuracy during the previous block.

Our main indices of behavior were accuracy and RTs. Accuracy scores were arcsine transformed $(2 \times$ arcsine square root $(x))$ to conform to parametric assumptions (Howell, 1997).

\section{Image Acquisition}

Participants were scanned at the Donders Centre for Cognitive Neuroimaging on a Siemens Tim Trio 3T Scanner using multiecho EPI (repetition time $=2.32$, echo times $=9,19.3,30$, and $40 \mathrm{msec}$, flip angle $=$ $\left.90^{\circ}\right)$. Each session consisted of $1360(4 \times 340$ scans per echo) scans of 38 slices (slice thickness: $2.5 \mathrm{~mm}$, resolution: $3.3 \mathrm{~mm} \times 3.3 \mathrm{~mm} \times 2.5 \mathrm{~mm})$. Thirty scans were acquired at the beginning of the first session to use as "weighing scans" (see fMRI Preprocessing section). Participants were scanned using a 32-channel head coil. Movement was prevented by positioning two cushions at either side of the participant's head and by placing tape across the forehead. Participants completed five sessions of the experimental task. A high-resolution structural scan of the brain was also acquired (MP-RAGE: 192 scans, repetition time $=2300 \mathrm{msec}$, echo time $=3.03 \mathrm{msec}$ ) to aid with preprocessing.

\section{fMRI Preprocessing}

Preprocessing and analysis were performed using SPM5. In line with established optimal procedures for analyzing multiecho data (Poser, Versluis, Hoogduin, \& Norris, 2006), data from the first echo was used to estimate six rigid body realignment parameters $(x, y, z$, roll, pitch, yaw) needed to align the images. The estimated realignment parameters were then used to align data from the other three echoes. Realignment was performed by aligning the first image of all the subsequent sessions to the first image of the first session and then realigning each image in each session to the first image in that session.

Images from the four echoes were then combined to a single image as a weighted-sum of data from the four echoes. Using data from the 30 weighing scans, a voxelspecific weighting between the four echoes was calculated using in-house software, which maximized the contribution of each echo according to its contrast-tonoise ratio (see Poser et al., 2006). The combined images were then slice-time corrected to the middle slice, coregistered with participant's structural MP-RAGE and then normalized to a standard template (Montreal Neuroimaging Institute $[\mathrm{MNI}]$ ) using the "unified segmentation" procedure (Ashburner \& Friston, 2005).

\section{Image Data Analysis}

Data were analyzed using the "summary statistics" approach (Mumford \& Nichols, 2009; Holmes \& Friston, 1998), in which contrast images from each participant are computed from running a separate general linear model (GLM) for each participant and then submitted to a (second-level) random effects analysis by running a second, group-level GLM.

The first-level design matrix was composed of a block diagonal matrix containing task-related and nuisance regressors for each session. The task regressors were formed from the onsets of initial encoding stimuli, "loss," "neutral," "win" screen, to-be-ignored stimuli, fixation cross (no-interference condition), to-be-updated stimuli and probe event, and end of block accuracy screen. In addition, parametric modulators (Buchel, Holmes, Rees, \& Friston, 1998), orthogonalized to the task regressor, were also entered for the three working memory conditions (ignore, no-interference, and update) regressors. These regressors corresponded to the valence of the received outcome on the current trial (1 for loss, 2 for neutral, and 3 for gain). This modeling approach was adopted because we were interested in the linear effects 
of outcome valence on signal during subsequent intervening stimuli. Finally, a parametric regressor corresponding to the $\mathrm{RT}$ for the probe event was also added. To efficiently model the BOLD response to the above experimental manipulations, all task-related regressors were convolved with a canonical hemodynamic response function.

Following Lund, Norgaard, Rostrup, Rowe, and Paulson (2005), 24 nuisance regressors were also included in the model: the six realignment parameters used to realign each image, the square of these realignment parameters, the first derivative of these realignment parameters, and the realignment parameters used to realign the previous volume (so as to account for spin-history effect, see Friston, Williams, Howard, Frackowiak, \& Turner, 1996). Data were also high-pass filtered (128 sec) to remove low-frequency signals from the data, and an AR(1) model was applied to adjust for serial correlations in the data. Microtime onsets were adjusted to take into account the earlier slice-timing correction.

This study aimed to investigate the effect of reward outcome, working memory demands, and their interaction. Accordingly, we generated the following contrast images at the first level and then examined group-level effects using separate one-sample $t$ tests at the second level.

i) Gain minus Loss (the "gain" screen regressor minus the "lose" screen regressor).

ii) Update minus Ignore (the to-be-updated stimuli minus the to-be-ignored stimuli).

iii) Outcome $\times$ Task interaction (the outcome-related parametric modulator of the update condition minus the outcome-related parametric modulator of the ignore condition).

\section{Connectivity Analysis}

The main aim of this study was to examine how reward signal in the ventral striatum affected working memory task-related neural (BOLD) responses. This question can most directly be addressed with a psychophysiological interaction (PPI) analysis (Friston et al., 1997). PPI can be used to examine the extent to which the coupling between a region (seed region) and other brain regions vary as a function of task context (psychological regressor). Here, we used the ventral striatum as a seed region. The ventral striatum was defined as comprising those voxels that were revealed to be active ( $p_{\text {uncorrected }}<$ .001) by the group-level gain minus loss contrast with a structural mask of the BG. The mask was generated using FSL FIRST toolbox (Patenaude, Smith, Kennedy, \& Jenkinson, 2011) to segment bilateral regions of participants' BG (nucleus accumbens, caudate nucleus, putamen, globus pallidus). The images from each participant were then combined by taking the sum of all voxels in each participant. To further specify the reward selectivity of these voxels, we removed from this seed region those voxels that were revealed to be also active $\left(p_{\text {uncorrected }}<.001\right)$ by the update minus ignore contrast. The voxels selected for the seed region were the same for all participants. From this seed region, the first eigenvariate was extracted from the BOLD signal time series for all voxels within that region. Given that these interactions occur at the neural level, the extracted BOLD signal was deconvolved into its expected neural components (Gitelman, Penny, Ashburner, \& Friston, 2003). The estimated "neural" response was then multiplied by a vector representing task context ( 1 for updating and -1 for ignore) to form the PPI term. This term was convolved with the canonical hemodynamic response function in SPM and entered as a regressor in the first-level GLM. The GLM also included the hemodynamic response function (HRF)-convolved "main effects" of task (a boxcar function-onsets and durations, as in the univariate analysis — with 1 for update events and -1 for ignore events) and the time course of the ventral striatum. Accordingly, the PPI focused on changes in connectivity that occur during the processing of intervening stimuli. Similar to our other model, the GLM also included 24 movement parameters as regressors of no interest.

The estimated beta values for the interaction term were passed to the second-level for random-effects analysis. Connectivity was assessed using a one-sample $t$ test. We examined two contrasts. First, we assessed regions that showed significantly greater ventral striatal connectivity during update (relative to ignore) and, second, regions that showed the reverse pattern (greater ventral striatal connectivity during ignore than update trials).

We also examined whether regions that showed a significant PPI overlapped with the ignore- or update-related regions. To this end, for ignore-related regions, we made a mask comprising regions that were significant $\left(p_{\text {uncorrected }}<\right.$ .001) for the ignore minus update contrast. Conversely, for update-related regions, we made a mask comprising regions that were significant $\left(p_{\text {uncorrected }}<.001\right)$ for the update minus ignore contrast.

\section{Brain-Behavior Correlations}

Brain-behavior relationships were examined in two complementary ways: regressing neural responses extracted from our ventral striatal ROI onto behavior and regressing our behavior of interest onto whole-brain neural responses.

First, an ANCOVA was used to assess the relationship between BOLD signal extracted from the ventral striatum, for the gain minus loss contrast, and working memory accuracy. For each participant, a beta value was extracted for the gain minus loss contrast (using MarsBAR; Brett, Anton, Valabregue, \& Poline, 2002). The beta values for each region were then $z$-scored before being entered as a covariate in an ANCOVA. Task (update, ignore) and Outcome (loss, gain) were entered as within-subject factors. 
Second, we entered our Outcome $\times$ Task interaction term for working memory accuracy ([ignore gain - ignore loss] - [update gain - update loss]) as a covariate into the whole-brain gain minus loss one-sample $t$ test (contrast (i)). The results of this analysis should corroborate our first brain-behavior analysis, but also extend it to other regions. As with the connectivity data, we examined whether there was overlap with ignore- and update-related regions.

\section{Statistical Thresholds}

When no ROI was used, clusters of signal were declared significant if the probability of obtaining a cluster of that extent across the whole-brain by chance was below $p<$ .05 using Guassian random field theory to control for family-wise error (FWE) rate (Friston, Worsley, Frackowiak, Mazziotta, \& Evans, 1994). An initial cluster-defining height threshold of $p_{\text {uncorrected }}<.001$ was used. The coordinates of the maxima of each cluster are reported in brackets. Voxel-wise statistical tests for these maxima are also reported in the Table 2. For contrasts where ROIs were specified, inferences about significance were made according to the intensity of each voxel's signal: Signals in voxels were deemed significant if the probability of observing a voxel at least as extreme as that observed was $p<.05$, corrected for comparisons across the ROI using random field theory. This was done to provide anatomical specificity and maintain sensitivity across small volumes.

\section{RESULTS}

\section{Behavioral Results}

For accuracy, there was a main effect of Task, $F(2,38)=$ $14.67, p<.001$, but no main effect of Outcome, $(F<1)$, or interaction between Task and Outcome, $F(4,76)=1.25$, $p=.29$. Post hoc tests revealed that accuracy differed between each pair of task conditions; participants were more accurate on update relative to ignore trials, $t(19)=$ $4.10, p<.001$, and no-interference trials, $t(19)=4.46$, $p<.001$, but there was no difference between ignore and no-interference trials, $t(19)=.556, p=.556$. See Table 1 for accuracy and RT data.
A similar analysis of RTs revealed a main effect of Task, $F(2,38)=23.66, p<.05$, no significant effect of Outcome, $F(2,38)=1.63, p>.05$, and no significant interaction between Task and Outcome, $F(4,76)=.58, p>$ .05. Post hoc tests revealed that participants showed higher RTs on ignore than on update trials $(p<.05)$ as well as higher RTs on no-interference than on both ignore $(p<.05)$ and update $(p<.05)$ trials.

Supplemental analyses of accuracy data confirmed that participants performed the task as intended. First, participants processed the intervening information in both the ignore and the update conditions. This was revealed by accuracy being higher for novel probe items than for distractor items, both in the ignore, $t(19)=2.37, p=$ .028 , and update conditions, $t(19)=2.76, p=.012$. This indicates that participants attended to the intervening stimuli in both conditions.

Second, participants were encoding the items presented initially at encoding in both conditions. Thus, there was no significant difference between performance on nointerference trials in the ignore block and that in the update block, $t(19)=.90, p=.378$. Furthermore, in the update condition, there was no significant difference in performance between trials with initially presented items (nontargets) and trials with target items, $t(19)=.62$, $p=.54$.

Finally, we also assessed whether the interaction between outcome and task varied as a function of Probe Type (novel, distracter or target). There was no significant two-way interaction between Task and Probe Type, F(2, $38)=1.32, p=.278$, or three-way interaction between Task, Outcome, and Probe Type, $F(4,76)=.325, p=$ 325. Thus reward did not induce a response bias.

\section{Imaging Data}

\section{Gain Minus Loss}

Contrasting the receipt of an unexpected gain with that of a loss revealed significant $(p<.05$, whole-brain FWEcorrected) clusters of increased BOLD signal in the ventral striatum $(x=14, y=14, z=-6)$ and the ventromedial $\mathrm{pFC}(x=-14, y=38, z=-12)$. No clusters were significant for the reverse contrast. Thus, consistent

Table 1. Accuracy (\%) and RTs (msec) for Ignore, No-interference (Ignore Block), No-interference (Update Block), and Update Blocks According to the Valence of the Preceding Received Outcome

\begin{tabular}{|c|c|c|c|c|c|c|}
\hline & \multicolumn{2}{|c|}{ Gain } & \multicolumn{2}{|c|}{ Neutral } & \multicolumn{2}{|c|}{ Loss } \\
\hline & Accuracy & $R T$ & Accuracy & $R T$ & Accuracy & $R T$ \\
\hline Ignore & $77(15)$ & $1014(202)$ & $80(12)$ & $992(195)$ & $78(11)$ & $1033(202)$ \\
\hline No-interference (Ignore block) & $80(13)$ & $1078(193)$ & $83(13)$ & 1063 (239) & $79(14)$ & $1079(250)$ \\
\hline No-interference (Update block) & $79(15)$ & $1078(241)$ & $82(15)$ & $1054(210)$ & $74(16)$ & $1043(223)$ \\
\hline Update & $84(12)$ & $972(202)$ & $87(7)$ & $936(192)$ & $88(8)$ & $947(174)$ \\
\hline
\end{tabular}

Standard deviations are presented in parentheses. 
Figure 2. Left: Percent signal change for the different outcomes (loss, neutral, and gain) in the ventral striatum (extracted from the cluster selected from the contrast gain minus loss, $p_{\text {uncorrected }}<.001$ ) Graph shows percent signal change [\%Signal Change (Beta $($ task $) \times \max ($ HRF $) \times 100) /$ (Beta (constant))] for each regressor calculated using rfxplot (Glascher, 2009). The error bar represents standard error of the difference between gains and losses. Right: An SPM of significant $(p<.05$ FWE whole-brain cluster-corrected) clusters for gains minus loss contrast. Increased BOLD

signal was found in the ventral striatum and the ventromedial $\mathrm{pFC}$ for the receipt of gains relative to losses.

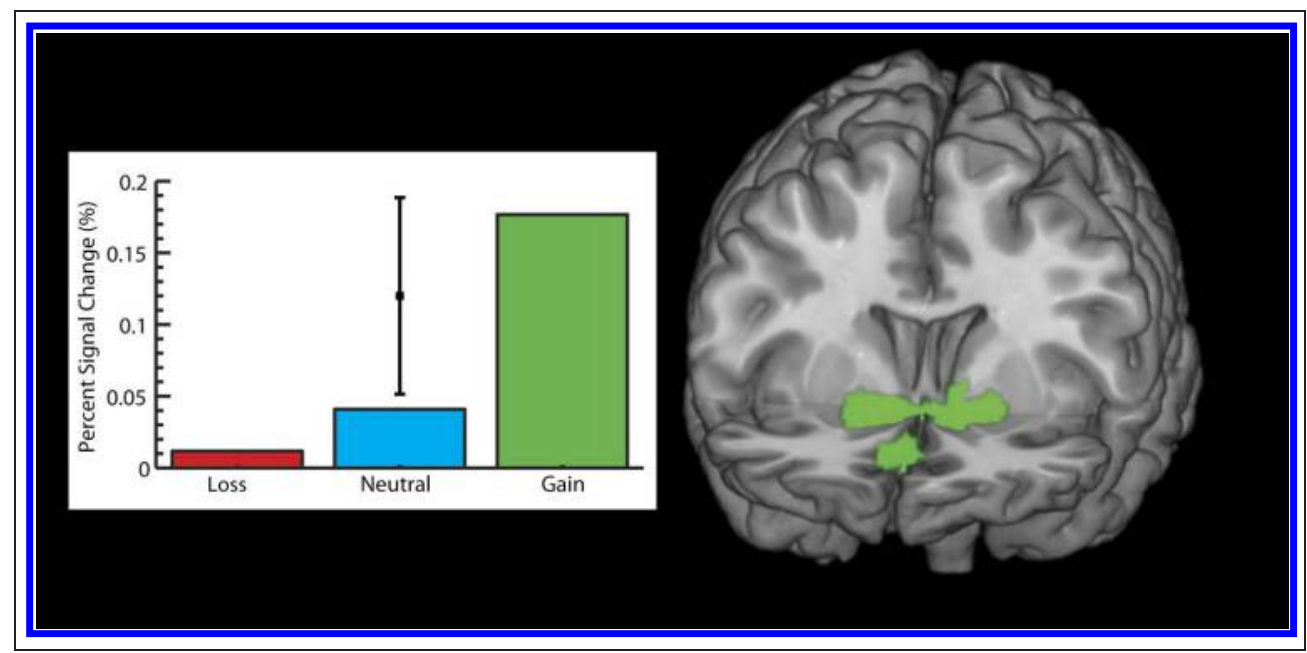

with our predictions, the ventral striatum showed greater BOLD signal during gain than loss (Figure 2). A full list of significant voxels for the whole brain is presented in Table 2.

\section{Update Minus Ignore}

Intervening stimuli that had to be used to update working memory relative to those that had to be ignored led to significantly ( $p<.05$, whole-brain FWE-corrected) increased BOLD signal in the left and right dorsal putamen (left: $x=20, y=10, z=4$, right: $x=22, y=14, z=4$ ), the left SMA, the right inferior gyrus $(x=50, y=6, z=$ $24)$, the right precentral gyrus $(x=50, y=6, z=24)$, the left and right fusiform gyri $(x=-36, y=-60, z=$ $-10)$, and a region near the left hippocampus $(x=-24$, $y=-24, z=4$ ). These regions are displayed in Figure 3 . The reverse contrast (ignore $>$ update) revealed significant clusters of BOLD signal in the left inferior parietal region $(x=-50, y=-52, z=40)$. Again, a full list of significant voxels is presented in Table 2 .

\section{Outcome $\times$ Task Interaction}

Contrasting the parametric effect of Outcome (gain, neutral, loss) in the update condition with that in the ignore condition did not produce any significant $(p<.05$ whole-brain FWE-corrected) clusters of increased or decreased BOLD signal.

\section{Connectivity Analysis}

Although there was no significant univariate modulation of Task processing by Outcome, such effects may be better captured by altered connectivity between rewardrelated and mnemonic areas. Accordingly, we conducted a PPI analysis to examine how connectivity with the rewardrelated ventral striatum (seed region) varied as a function of working memory task demands. No regions had significantly greater connectivity with the ventral striatum in the update than the ignore conditions, even when the statistical threshold was lowered to $p_{\text {uncorrected }}<.001$. However, the reverse contrast, reflecting greater connectivity with the ventral striatum in the ignore than the update conditions, revealed significant $(p<.05$, wholebrain FWE-corrected) clusters in the left dorsolateral $\mathrm{pFC}$ $(x=-40, y=6, z=42)$, the right dorsolateral $\mathrm{pFC}$ $(x=44, y=20, z=40)$, the dorsomedial pFC $(x=8$, $y=36, z=44)$, the left anterior pFC $(x=-42, y=50$, $z=2)$, and the left TPJ ( $x=-56, y=-46, z=28)$. These regions are shown in Figure 4 . Thus, BOLD signal in the reward-related ventral striatum showed higher correlations with prefrontal and parietal regions during ignore trials than update trials.

Next we examined overlap between regions showing differential connectivity with the ventral striatum as function of task context and regions sensitive to the main effect of task (ignore versus update; $p_{\text {uncorrected }}=.001$ ). Five of the five regions identified in the PPI contrast were present in the ignore $>$ update contrast. All regions except the dorsomedial pFC survived correction for multiple comparisons with the entire search volume of the ignore minus update contrast. None of the voxels identified in the PPI contrast were significant $(p<.05$, FWE voxel-level small volume-corrected) within an inclusive mask of update-related $\left(p_{\text {uncorrected }}<.001\right.$, update $>$ ignore contrast) regions. This shows that there is a strong correspondence between prefrontal and parietal regions preferentially involved in ignoring irrelevant information and those regions modulated by ventral striatal BOLD signal in a task-dependent manner. Thus, signal in the rewardrelated ventral striatum was associated with signal in regions 
Table 2. BOLD Signal Peaks (Voxelwise $p<.001$, Cluster Extent $>15$ ) for Main Contrasts of Interest, Shown at a More Liberal Threshold for Illustrative Purposes

\begin{tabular}{|c|c|c|c|c|c|c|}
\hline Contrast & Label & $\begin{array}{l}\text { Cluster } \\
\text { Extent }\end{array}$ & $t$ & $\begin{array}{c}\text { p Value } \\
\text { (FWE Whole-brain } \\
\text { Cluster-corrected) }\end{array}$ & $\begin{array}{c}p \text { Values } \\
\text { (FWE Whole-brain, } \\
\text { Voxel Level) }\end{array}$ & $\begin{array}{c}M N I \\
\text { Coordinates } \\
(x, y, z)\end{array}$ \\
\hline \multirow[t]{20}{*}{ UPDATE > IGNORE } & Left fusiform gyrus & 14700 & 11.40 & .000 & .000 & $-36-60-10$ \\
\hline & Left putamen & 3353 & 9.18 & .000 & .002 & -20104 \\
\hline & Right inferior frontal & 428 & 7.97 & .000 & .012 & 50624 \\
\hline & Left supplementary motor area & 888 & 6.80 & .000 & .071 & -4266 \\
\hline & Right putamen & 353 & 6.44 & .000 & .122 & 22144 \\
\hline & Optic radiation tract & 136 & 5.58 & .05 & .412 & $-24-24-4$ \\
\hline & Left precuneal white matter & 96 & 5.24 & .150 & .607 & $-24-4032$ \\
\hline & Left paracentral gyrus & 52 & 5.05 & .510 & .723 & $-12-3874$ \\
\hline & Left inferior gyrus & 82 & 5.03 & .223 & .730 & -463412 \\
\hline & Left superior orbital gyrus & 28 & 4.93 & .853 & .791 & $-1632-14$ \\
\hline & BA 6 & 198 & 4.83 & .010 & .841 & $36-250$ \\
\hline & Left precentral gyrus & 67 & 4.77 & .340 & .870 & $-20-2074$ \\
\hline & Right precuneal white matter & 17 & 4.76 & .964 & .871 & $32-4820$ \\
\hline & Left Heschl's gyrus & 22 & 4.55 & .923 & .944 & $-30-3014$ \\
\hline & Right ventral insula & 37 & 4.46 & .725 & .966 & $42-6-20$ \\
\hline & Right superior temporal gyrus & 16 & 4.39 & .970 & .977 & $66-10-2$ \\
\hline & Right supplementary motor area & 19 & 4.31 & .949 & .987 & $10-1076$ \\
\hline & Left superior temporal gyrus & 19 & 4.09 & .949 & .998 & $-58-3812$ \\
\hline & Right rolandic operculum & 21 & 4.03 & .932 & .999 & -5282 \\
\hline & Left superior frontal gyrus & 15 & 3.99 & .976 & .999 & $-24-454$ \\
\hline \multirow[t]{5}{*}{ IGNORE $>$ UPDATE } & Left angular gyrus & 82 & 4.97 & .223 & .769 & $-38-7446$ \\
\hline & Left inferior parietal lobule & 336 & 4.81 & .001 & .851 & $-50-5240$ \\
\hline & Left middle orbital gyrus & 23 & 4.31 & .913 & .986 & $-4046-4$ \\
\hline & Left middle frontal gyrus & 41 & 4.25 & .666 & .991 & -362044 \\
\hline & Left superior medial gyrus & 16 & 4.14 & .970 & .996 & -43646 \\
\hline \multirow[t]{6}{*}{ GAIN $>$ LOSS } & Left ventral striatum & 460 & 7.28 & .000 & .029 & $-1438-12$ \\
\hline & Right ventral striatum & 1125 & 6.97 & .0000 & .047 & $1414-6$ \\
\hline & Posterior cingulate & 117 & 5.71 & .113 & .313 & $2-3438$ \\
\hline & left inferior parietal lobe & 45 & 4.70 & .646 & .863 & $-52-4646$ \\
\hline & Left middle occipital gyrus & 17 & 4.23 & .963 & .986 & $-28-6432$ \\
\hline & Left superior frontal gyrus & 39 & 4.10 & .726 & .995 & -161462 \\
\hline LOSS $>$ GAIN & No significant $(p<.001)$ activation & & & & & \\
\hline \multirow{2}{*}{$\begin{array}{l}\text { Parametric reward } \\
\text { effect UPDATE > } \\
\text { Parametric reward } \\
\text { effect IGNORE }\end{array}$} & Right globus pallidus & 28 & 6.57 & .831 & .118 & $122-2$ \\
\hline & Left middle cingulate & 36 & 4.67 & .697 & .940 & -16242 \\
\hline $\begin{array}{l}\text { Parametric Reward } \\
\text { effect IGNORE > } \\
\text { Parametric Reward }\end{array}$ & No significant activation & & & & & \\
\hline
\end{tabular}


Figure 3. Left: An SPM of significant $(p<.05$ FWE wholebrain cluster-corrected) clusters for update minus ignore trials. Increased BOLD signal was found in the left dorsal striatum during update relative to ignore trials. A complete list of regions can be found in Table 2. Right: Graph shows percent signal change for the ignoring, updating, and no-interference conditions in the dorsal striatum (extracted from the cluster selected from the contrast updating minus ignoring, $p_{\text {uncorrected }}<.001$ ) The error bar represents the standard error of the difference between updating and ignoring.

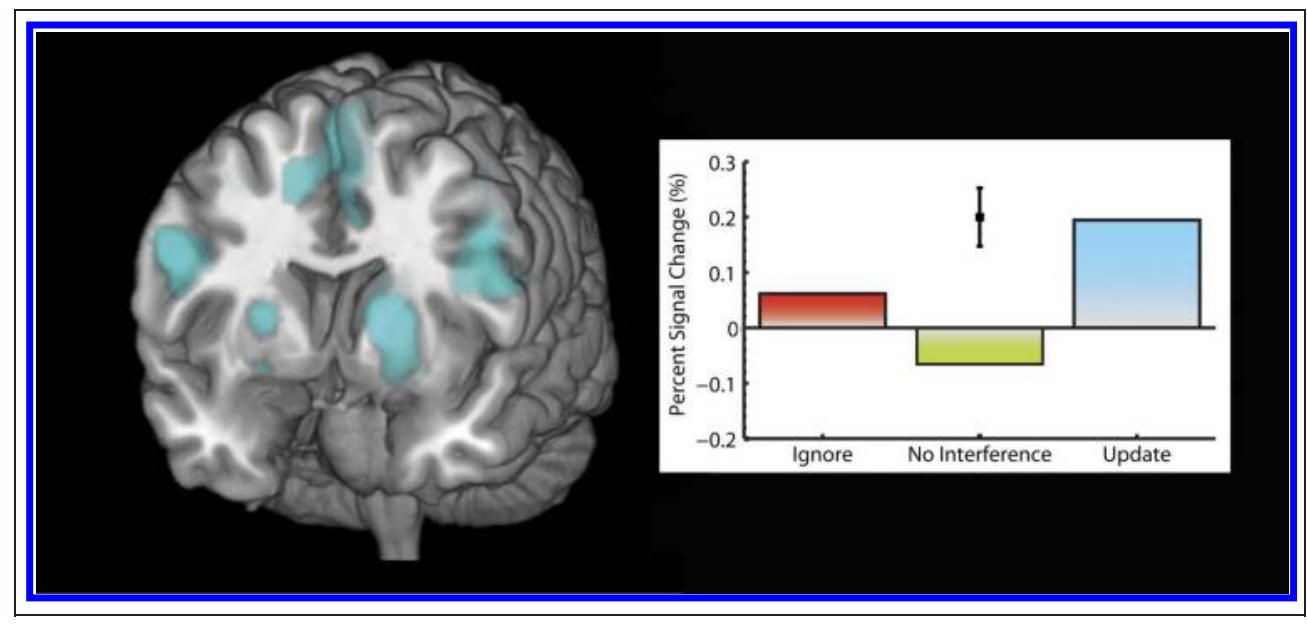

that support distractor resistance but not in regions that support updating of working memory representations, including the (dorsal) striatum.

\section{Brain-Behavior Relationships}

As stated above, there was no overall main effect of reward on working memory performance. However, further analyses revealed significant brain-behavior correlations. Specifically, the effect of reward on distractor resistance versus updating (in terms of accuracy) correlated with the effect of reward on BOLD signal, already at the time of outcome. This was confirmed by two different analyses. First, we performed a repeated-measures ANCOVA on participant's accuracy scores with Task (update and ignore) and Outcome (gain loss) as withinsubject factors and inserted reward-related Ventral Striatal Signal ( $z$-score beta values for the gain minus loss contrast) as a covariate. There was a significant three-way interaction between Ventral Striatal Signal, Task, and Outcome, $F(1,18)=9.44, p=.008$. This indicates that the effect of reward on working memory performance varied according to the magnitude of gain-induced BOLD signal in the ventral striatum. Regression analysis was used to unpack this significant three-way interaction. This significant three-way interaction was the result of two strong trends in the opposite direction: a near-significant trend for a positive correlation between ventral striatal rewardrelated response and accuracy on gain minus loss ignore trials (standardized beta $=.44, p=.052$ ), and a trend for a negative correlation between ventral striatal rewardrelated response and accuracy on gain minus loss update trials (standardized beta $=-.42, p=.068$ ). None of the other two-way interactions were significant, and there was no significant relationship between ventral striatal BOLD signal and gain minus loss performance in the nointerference condition $(r=.10, p=.66)$.

Second, the above analysis was corroborated by a whole-brain analysis of outcome-related signal, in which the effect of reward on working memory performance (ignore vs. update accuracy) was used as a covariate of interest. Given the above analysis, we examined the positive association between the covariate and BOLD signal, that is, areas that were positively associated with higher
Figure 4. An SPM of voxels that showed significantly $(p<0.05$ FWE whole-brain cluster-corrected) higher connectivity between the ventral striatum during ignore, relative to update trials. Left $=$ left hemisphere; Right $=$ right hemisphere.

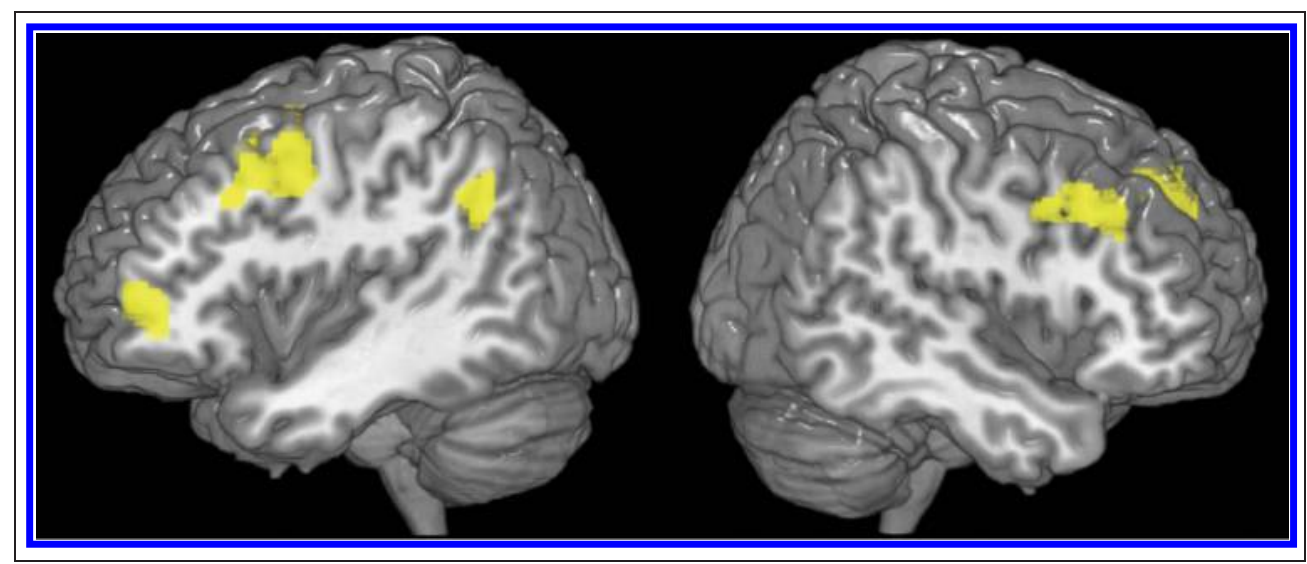


Figure 5. (A) SPM showing regions that showed a significant $(\mathrm{FWE}<0.05$ whole-brain cluster-corrected) relationship with the behavioral interaction between outcome and working memory for the gain minus loss contrast. Higher BOLD signal in these regions was associated with a greater interaction between outcome and working memory. (B) A breakdown of the association between performance (accuracy on gain relative to loss trials) and BOLD signal for gain minus loss events according to task. Areas that correlate positively with accuracy on gain relative to loss trials on ignore blocks are shown in red. For ignore trials, the left ventral striatum, left dorsolateral $\mathrm{pFC}$, left frontopolar cortex, and right inferior frontal cortex survived correction for multiple comparisons (FWE $<0.05$, small volume-corrected). Areas that correlative negatively with accuracy on gain relative to loss trials are shown in green. On update trials, the left frontopolar cortex and right inferior frontal cortex survived correction for multiple comparisons (FWE $<0.05$, small volume-corrected). For illustrative purpose, the above images display significant areas at a more liberal threshold ( $p<.001$, uncorrected). accuracy on gain minus loss trials for ignore relative to the update conditions. As can be seen in Figure 5 and consistent with the above-mentioned ANCOVA, clusters of significant ( $p<.05$, whole-brain FWE-corrected) signal were observed in the left ventral striatum. In addition, significant brain-behavior correlations were also found in the right inferior frontal cortex $(x=56, y=24, z=-2)$, the left frontopolar cortex $(x=-32, y=50, z=-2)$, and the left dorsolateral pFC $(x=-36, y=12, z=52)$. Follow-up analyses decomposed these interactions by examining the brain-behavior correlations separately for ignore and update conditions. The left ventral striatum, left dorsolateral $\mathrm{pFC}$, left frontopolar cortex, and right inferior frontal cortex were correlated positively significantly $(p<$ .05 , small volume FWE-corrected) with the relative increase in accuracy on gain relative to loss trials in the ignore condition (Figure 5A). In contrast, the left frontopolar cortex and right inferior frontal cortex correlated negatively ( $p<.05$, small volume FWE-corrected) with the relative increase in accuracy on gain relative to loss trials in the update condition (Figure 5B). There was a trend toward a significant negative correlation with the left ventral striatum ( $p=.07$, FWE small volume voxel-level correction).

We also examined the overlap between regions modulated by accuracy on gain minus loss trials (those regions shown in Figure 5A) and regions modulated by task (ignore, update). The right inferior, left frontopolar, and left dorsolateral pFC were all significant $(p<.05$, small volume FWE-corrected) when masked inclusively by the ignore-related pFC regions. However, when masked inclusively by the update-related regions (which include the dorsal striatum), no regions were significant ( $p<.05$, small volume FWE-corrected). Together, these results show that reward-related signal in the ventral striatum and in $\mathrm{pFC}$ regions were associated with rewardinduced increases in accuracy on ignore relative to update trials. Thus reward-induced increases in ventral striatal and prefrontal BOLD signal were associated with taskspecific improvement in distractor resistance (relative to updating). A summary of all the imaging results from this study is presented in Figure 6.

\section{DISCUSSION}

This study provides evidence that reward-related signals interact with the pFC to enhance distractor resistance of working memory representations. This observation generally concurs with previous studies that have found that reward and working memory interact in the frontal cortex (Krawczyk \& D’Esposito, 2013; Marquand et al., 2011; Dolcos, Diaz-Granados, Wang, \& McCarthy, 2008; Krawczyk et al., 2007) but critically extends this prior work by showing that the enhancing effect of reward on prefrontal function is task specific, that is, reward-related signals have opposite effects on distractor resistance and updating.

Consistent with previous studies, increased BOLD signal was found in the ventral striatum for receipt of an unexpected financial gain relative to unexpected financial loss (Elliott, Newman, Longe, \& Deakin, 2003; Berns, McClure, Pagnoni, \& Montague, 2001; Delgado et al., 2000). Thus, this study was successful in eliciting the ventral-striatal reward response known to correlate with dopamine release (Jenkins, 2012; Glimcher, 2011; Schott et al., 2008; Knutson \& Gibbs, 2007). Over all participants there was no significant interaction between outcome and task in terms of behavior. However, in line with hypotheses generated from neurocomputational models (Durstewitz \& Seamans, 2008; Hazy et al., 2007), the magnitude of 
the response in the ventral striatum and $\mathrm{pFC}$ was related to working memory accuracy in a task-dependent manner. We found that increased BOLD signal in the ventral striatum and $\mathrm{pFC}$ during the receipt of gains relative to losses had opposite effects on ignoring and updating items in working memory; greater BOLD signal in the ventral striatum and $\mathrm{pFC}$ was associated with enhanced accuracy on ignore trials but reduced accuracy on update trials. In contrast, we found no evidence for any such modulation in the dorsal striatum. Given the association between unexpected reward and dopamine (Glimcher, 2011; Schott et al., 2008), it is likely that the observed increase in BOLD reflects increased dopamine levels. Thus, the receipt of a gain, relative to a loss, induced changes in the $\mathrm{pFC}$ that were conducive to filtering out irrelevant information from working memory but detrimental for flexibly updating items in working memory.

Our univariate analysis failed to reveal any regions that were differentially modulated by the preceding outcome in a task-dependent manner. Rather, we found that the interaction between outcome and task was accompanied by differential coupling between the ventral striatum and pFC. Thus the ventral striatum was found to be differentially connected with ignore- versus update-related regions of the $\mathrm{pFC}$; connectivity was higher during ignore trials than update trials. Accordingly, the ventral striatum was coupled to regions of the pFC that were responsible for filtering out irrelevant information from entering mnemonic networks. Therefore, an increased response

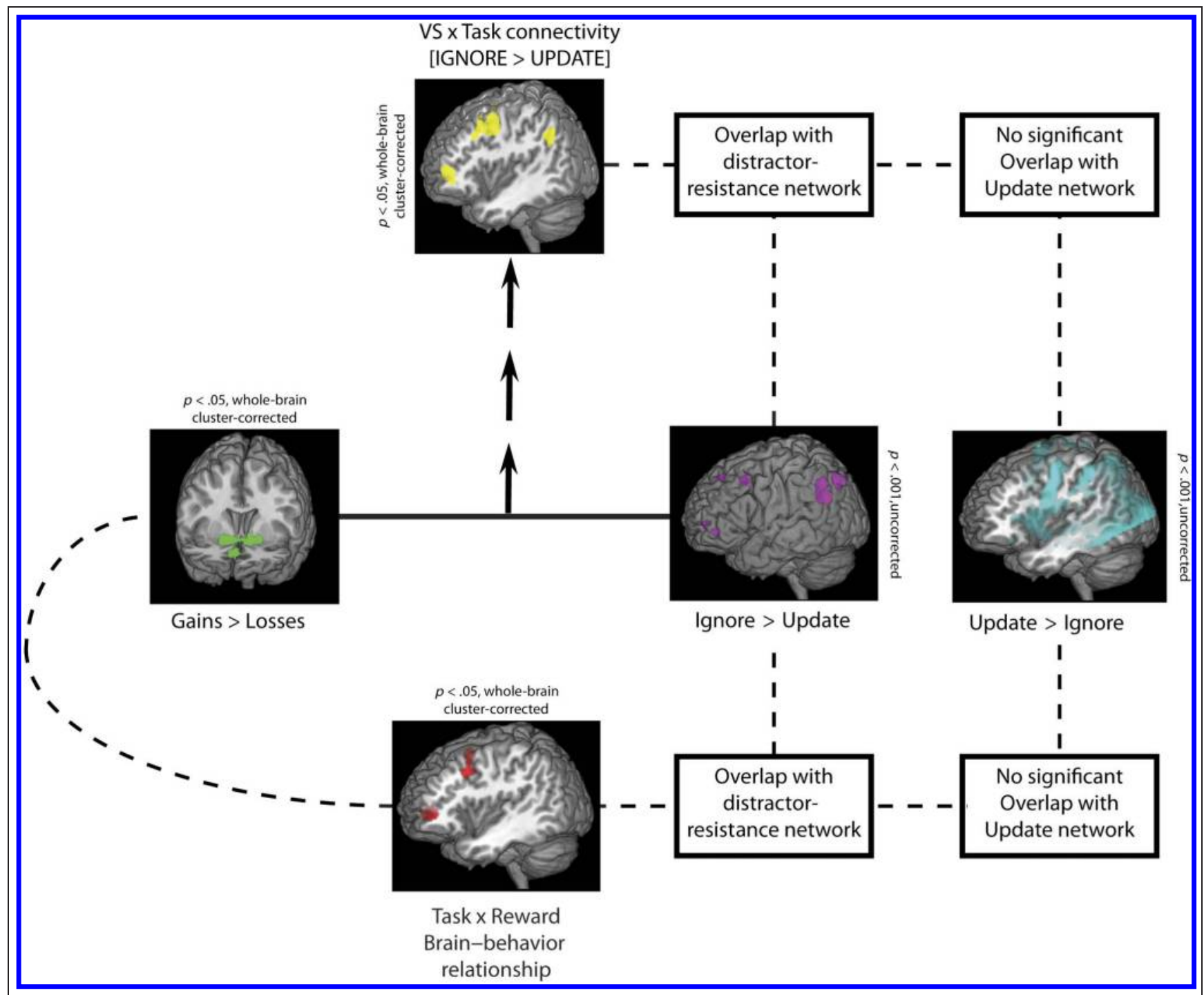

Figure 6. A summary of the results presented in this study. The receipt of gains relative to losses led to increased BOLD signal in the ventral striatum. The extent to which there was an interaction between working memory and reward was related to the magnitude of the BOLD signal changes in the ventral striatum, left dorsolateral pFC, and left frontopolar cortex (Figure 5A). In addition there was increased connectivity between the ventral striatum and the left dorsolateral $\mathrm{pFC}$ and frontopolar cortex during ignore relative to update trials. Both regions that showed a brain-behavior interaction and regions that showed task-based connectivity with the VS were present within the distracter resistance network (Ignore $>$ Update) but not the update network (Update > Ignore). 
to gains, relative to losses, in the ventral striatum was associated with increased recruitment of the distractor resistance network. Again, no such modulation of the dorsal striatum was observed. Consistent with this hypothesis are the results from our brain-behavior analysis, showing that signal in the distractor resistance network is associated with different behavioral effects during the two task conditions. During ignore trials, increased signal within the distractor resistance network is associated with enhanced performance, whereas during updating, increased signal of this network is associated with impaired performance (Figure 5). Therefore, it seems that, in the context of this experiment, gains promote the expression of the psychological and neural mechanisms responsible for distractor resistance, leading to differential effects on ignoring and updating. Cumulatively, therefore, it seems that both gains and gain-related ventral striatum signal act on the pFC, possibly via corticostriatalthalamic loops, to promote distractor resistance but to impair updating.

These results have implications of neurocomputational models of cognitive control and our understanding of psychiatric disorders. First, with regard to computational theories, these results are generally consistent with the predictions of recent computational theories of prefrontal dopamine (Durstewitz \& Seamans, 2002, 2008; Durstewitz, Seamans, \& Sejnowski, 2000). According to these models, dopamine modulates the gain of prefrontal neurons through its actions on numerous different ion (e.g., NMDA, $\mathrm{Ca}^{2+}$ ) channels (Seamans \& Yang, 2004; Servan-Schreiber et al., 1990). Aggregation of knowledge about the effects of dopamine $\mathrm{D}_{1}$ and $\mathrm{D}_{2}$ receptor stimulation on these intracellular processes has led to the proposal that they can give rise to two different states: a D1 and a D2 state (Durstewitz \& Seamans, 2002, 2008; Durstewitz et al., 2000). The pFC is argued to occupy a D2 state at either low or high dopamine levels. This state supports neuronal ensembles with little differentiation between the activity of neurons that represent relevant and irrelevant information. Thus, because relevant information is less robustly represented, flexibility is enhanced (cognitive flexibility). However, at intermediate dopamine levels, a D1 state exists. In this state, there is a sharp demarcation between the activity of neurons that represent relevant and irrelevant information. This enables relevant information to be robustly maintained in the face of distraction (cognitive stability) but, as a corollary, reduces the flexibility of these neuronal ensembles. The putatively increased dopamine in the ventral striatum and $\mathrm{pFC}$ after a gain, relative to a loss, in this study may have shifted the pFC from a D2 into a D1 state (Durstewitz \& Seamans, 2008), which manifested itself as altered coupling between the ventral striatum and ignore-related regions in the pFC. However, this hypothesis remains to be tested in future work, not least because the type of connectivity analyses employed here does not allow us to draw definitive conclusions about the direction of the relationship between the $\mathrm{pFC}$ and ventral striatum.

The hypotheses for this experiment were leveraged from knowledge about the role of dopamine in reward and working memory. Of course, definitive evidence for a role of dopamine in the effects observed here must come from future psychopharmacological or quantitative PET studies. More generally, our effects are consistent with the known effects of reward on working memory obtained using a variety of methodologies, indicating that reward enhances the representation of relevant information across a variety of timescales (Murty \& Adcock, in press; Kennerley \& Wallis, 2009b; Chumbley, Dolan, \& Friston, 2008; Krawczyk et al., 2007; Adcock, Thangavel, Whitfield-Gabrieli, Knutson, \& Gabrieli, 2006; Wittmann et al., 2005; though see Krawczyk \& D'Esposito, 2013). The novelty of this study's finding is the task specificity of the enhancing effects of reward-related signals in the ventral striatum and $\mathrm{pFC}$, which are associated with enhanced distractor resistance but impaired updating.

In contrast to the ventral striatum, the dorsal striatum had a purely mnemonic role. Numerous theoretical and computational accounts of the striatum have argued that this region plays a key role in controlling the contents of working memory (Hazy et al., 2007; Gruber, Dayan, Gutkin, \& Solla, 2006). These contentions have been supported by empirical work that has found that the striatum is necessary for ignoring distracting information (Baier et al., 2010; Collins et al., 2000) as well as for updating mnemonic representations (Murty et al., 2011; Roth, Serences, \& Courtney, 2006). The results of this study help us further delineate and characterize the role of the dorsal striatum in working memory. Mnemonic task demands preferentially modulated BOLD signal in the dorsal striatum. Specifically, BOLD signal in the dorsal striatum was increased, relative to the no-interference condition, in both the ignore and update conditions (Figure 3). However, dorsal striatal BOLD signal was increased to a greater extent when novel stimuli had to be encoded into working memory than when they had to be ignored. This finding extends our knowledge of the dorsal striatum by demonstrating that its role in working memory can be dichotomized according to whether new information has to be allowed or prevented from entering mnemonic networks.

Recent neurocomputational models have highlighted a common role for dopamine in the striatum in, on the one hand, reward versus punishment processing and, on the other hand, updating and distractor resistance of working memory (Maia \& Frank, 2011; Frank, 2005). As such, it is surprising that we did not observe any effects of rewardon task-related responses in the (update-related dorsal) striatum. Similarly, there were no reward-induced changes in working memory task-related connectivity between the ventral and dorsal striatum, an observation that could have been anticipated based on anatomical evidence for dopamine-dependent connections between ventral and 
dorsal striatum (Haber, 2003; Haber, Fudge, \& McFarland, 2000). We believe that this failure to find reward modulation of striatal responses during working memory is unlikely to reflect suboptimal task design or statistical power, because we did observe highly significant rewardrelated signal in the ventral striatum as well as highly significant update-related signal in the dorsal striatum. By contrast, there were no effects of reward on update-related signal in the striatum, even at a lower statistical threshold. Although the present results could be seen as evidence against the computational models that give a prominent role to the striatum in cognitive control (e.g., Hazy et al., 2007; O’Reilly \& Frank, 2006), several qualifications are needed. First, it should be noted that the coupling between ventral striatum and $\mathrm{pFC}$ is indeed consistent and could have been anticipated by the O'Reilly and Frank (2006) model, given that in this model the ventral striatum represents the reward value of items maintained in $\mathrm{pFC}$ that contribute to task success. Therefore, in this respect the present results support those models. Second, the failure to find an interaction between some aspect of reward and the dorsal striatum could reflect several factors, such as the nature of the updating required by this task. In this experiment, a total update design was employed, which required nonselective updating of all items. A key feature of computational models of the BG's role in working memory is that they facilitate the selective updating of mnemonic representations (Hazy et al., 2007), a distinction supported by empirical findings (Murty et al., 2011). Therefore, effects of reward on the dorsal striatum may be confined to instances where information has to be selectively updated, for example, only changing the color of the remembered shapes. Alternatively, the failure to find a modulation of dorsal striatal BOLD signal by reward during updating may reflect the possibility that dopamine in the dorsal striatum is only important for the ability to acquire (learn) when to update, something which may only occur in the first few trials.

Finally, an alternative mechanism by which reward might have altered distractor resistance versus updating is by reinforcement of the target cues being maintained and could theoretically explain the pattern observed here. This alternative reinforcement account seems less likely, however, because gains altered neural signals in the distractor resistance but not updating network during subsequent intervening stimuli. Nevertheless, in future studies, it will be interesting to assess whether reward acts on the previously encoded information or the upcoming intervening information, for example, by manipulating the delay periods between encoding and reward receipt and between reward receipt and intervening stimuli.

An important feature of the paradigm used here is the fact that the outcome was delivered during the delay period. This had the dual benefit of enabling us to examine the neurocognitive processes of interest and in removing the potentially confounding effects of differential motivation at the point of encoding in the different outcome conditions. It also allowed us to examine the effects of putative dopamine release during the delay period, associated with unexpected reward, on mnemonic networks. Our paradigm could be criticized on the grounds that it is unnatural to receive a reward/loss during the performance of a specific task. However, in the modern world, we are increasingly bombarded by reward-related information during cognitive processing. For example, when performing some task on our computer, we may receive some good or bad news by email (paper accepted/paper rejected). The receipt of this news is likely to impact our cognitive functioning irrespective of whether the email is related to our current task. However, the results of this study suggest that good (or bad) news will influence subsequent neurocognitive processing in a task-specific manner and may enhance or diminish those processes according to the task being performed. Furthermore, the interaction between reward processing and task is likely to vary as a function of task difficulty and the perceived magnitude of the reward. This question should be address in future research.

The finding that unexpected rewards can impact working memory performance has important implications for understanding cognitive dysfunction in disorders that are accompanied by reward-related deficits. For example, the present findings suggest that if reward-related signals are attenuated, as might be the case in addiction (Goldstein et al., 2007; Volkow et al., 2007), then this may account for some of the working memory deficits observed in these groups (Tomasi et al., 2007). Interestingly, because impaired working memory is related to impaired decisionmaking in people with substance dependence (Bechara \& Martin, 2004), this may lead to cycles of dependence and relapse that often characterize this disorder. Similarly, Parkinson's disease has also been associated with deficits in reward processing and working memory, in particular filtering out irrelevant information. Thus, given this study's finding that reward-related response guide working memory (Rowe et al., 2008; Cools, Altamirano, \& D’Esposito, 2006), working memory deficits in PD may be caused by deficits in reward processing. Future experiments should evaluate this hypothesis.

\section{Acknowledgments}

We thank Sharissa Corporaal for help with data acquisition. This work was conducted in the context of a VIDI grant from the Innovational Research Incentives Scheme of the Netherlands Organisation for Scientific Research to R. C.; a Human Frontiers Science Program grant to Kae Nakamura, Nathaniel Daw, and R. C.; and a James McDonnell Scholar Award to R. C. R. C. is a consultant to Abbott Laboratories, but she is not an employee or a stock shareholder.

Reprint requests should be sent to Sean James Fallon, Centre for Cognitive Neuroimaging, Donders Institute for Brain, Cognition and Behaviour, Radboud University Nijmegen, Kapittelweg 29, 6500 HB, Nijmegen, The Netherlands, or via e-mail: s.fallon@ donders.ru.nl. 


\section{REFERENCES}

Aarts, E., van Holstein, M., \& Cools, R. (2011). Striatal dopamine and the interface between motivation and cognition. Frontiers in Psychology, 2, 163

Adcock, R. A., Thangavel, A., Whitfield-Gabrieli, S., Knutson, B., \& Gabrieli, J. D. (2006). Reward-motivated learning: Mesolimbic activation precedes memory formation. Neuron, 50, 507-517.

Ashburner, J., \& Friston, K. J. (2005). Unified segmentation. Neuroimage, 26, 839-851.

Baier, B., Karnath, H. O., Dieterich, M., Birklein, F., Heinze, C., \& Muller, N. G. (2010). Keeping memory clear and stableThe contribution of human basal ganglia and prefrontal cortex to working memory. Iournal of Neuroscience, 30, 9788-9792.

Bechara, A., \& Martin, E. M. (2004). Impaired decision making related to working memory deficits in individuals with substance addictions. Neuropsychology, 18, 152-162.

Berns, G. S., McClure, S. M., Pagnoni, G., \& Montague, P. R. (2001). Predictability modulates human brain response to reward. Journal of Neuroscience, 21, 2793-2798.

Brainard, D. H. (1997). The Psychophysics Toolbox. Spatial Vision, 10, 433-436.

Braver, T. S., \& Barch, D. M. (2002). A theory of cognitive control, aging cognition, and neuromodulation. Neuroscience and Biobehavioral Reviews, 26, 809-817.

Braver, T. S., \& Cohen, J. D. (2000). On the control of control: The role of dopamine in regulating prefrontal function and working memory. In L. Stephen Mousell \& John Driver (Eds.), Control of cognitive processes: Attention and performance XVIII (pp. 713-737). Cambridge, MA: MIT Press.

Brett, M., Anton, J.-L., Valabregue, R., \& Poline, J.-B. (2002). Region of interest analysis using the MarsBar toolbox for SPM 99. Neuroimage, 16, S497.

Buchel, C., Holmes, A. P., Rees, G., \& Friston, K. J. (1998). Characterizing stimulus-response functions using nonlinear regressors in parametric fMRI experiments. Neuroimage, 8 , 140-148.

Chao, L. L., \& Knight, R. T. (1995). Human prefrontal lesions increase distractibility to irrelevant sensory inputs. NeuroReport, 6, 1605-1610.

Chumbley, J. R., Dolan, R. J., \& Friston, K. J. (2008). Attractor models of working memory and their modulation by reward Biological Crbernetics, 98, 11-18.

Collins, P., Wilkinson, L. S., Everitt, B. J., Robbins, T. W., \& Roberts, A. C. (2000). The effect of dopamine depletion from the caudate nucleus of the common marmoset (Callithrix jacchus) on tests of prefrontal cognitive function. Behavioral Neuroscience, 114, 3-17.

Cools, R., Altamirano, L., \& D'Esposito, M. (2006). Reversal learning in Parkinson's disease depends on medication status and outcome valence. Neuropsvchologia, 44, $1663-1673$

Cools, R., \& D'Esposito, M. (2011). Inverted-U-shaped dopamine actions on human working memory and cognitive control. Biological Psychiatry, 69, e113-e125.

Delgado, M. R., Nystrom, L. E., Fissell, C., Noll, D. C., \& Fiez, J. A. (2000). Tracking the hemodynamic responses to reward and punishment in the striatum. Lournal of Neuropbysiology 84, 3072-3077.

Dickinson, A., \& Balleine, B. (1994). Motivational control of goal-directed action. Animal Learning \& Behavior, 22, $1-18$.

Dolcos, F., Diaz-Granados, P., Wang, L., \& McCarthy, G. (2008) Opposing influences of emotional and non-emotional distracters upon sustained prefrontal cortex activity during a delayed-response working memory task. Neuropsvchologia 46, 326-335.

Durstewitz, D., \& Seamans, J. K. (2002). The computational role of dopamine D1 receptors in working memory. Neural Networks, 15, 561-572.

Durstewitz, D., \& Seamans, J. K. (2008). The dual-state theory of prefrontal cortex dopamine function with relevance to catechol-o-methyltransferase genotypes and schizophrenia. Biological Psvchiatrv, 64, 739-749.

Durstewitz, D., Seamans, J. K., \& Sejnowski, T. J. (2000). Dopamine-mediated stabilization of delay-period activity in a network model of prefrontal cortex. Journal of Neurophysiology, 83, 1733-1750.

Elliott, R., Newman, J. L., Longe, O. A., \& Deakin, J. F. (2003). Differential response patterns in the striatum and orbitofrontal cortex to financial reward in humans: A parametric functional magnetic resonance imaging study. Journal of Neuroscience, 23, 303-307.

Frank, M. J. (2005). Dynamic dopamine modulation in the basal ganglia: A neurocomputational account of cognitive deficits in medicated and nonmedicated Parkinsonism. Journal of Cognitive Neuroscience, 17, 51-72.

Frank, M. J., \& O’Reilly, R. C. (2006). A mechanistic account of striatal dopamine function in human cognition: Psychopharmacological studies with cabergoline and haloperidol. Behavioral Neuroscience, 120, 497-517.

Friston, K. J., Buechel, C., Fink, G. R., Morris, J., Rolls, E., \& Dolan, R. J. (1997). Psychophysiological and modulatory interactions in neuroimaging. Neuroimage, 6, 218-229.

Friston, K. J., Williams, S., Howard, R., Frackowiak, R. S., \& Turner, R. (1996). Movement-related effects in fMRI time-series. Magnetic Resonance in Medicine, 35, 346-355.

Friston, K. J., Worsley, K. J., Frackowiak, R., Mazziotta, J. C., \& Evans, A. C. (1994). Assessing the significance of focal activations using their spatial extent. Human Brain Mapping. 1, 210-220.

Funahashi, S., Bruce, C. J., \& Goldman-Rakic, P. S. (1993). Dorsolateral prefrontal lesions and oculomotor delayedresponse performance: Evidence for mnemonic "scotomas". Journal of Neuroscience, 13, 1479-1497.

Gerfen, C. R., \& Surmeier, D. J. (2011). Modulation of striatal projection systems by dopamine. Annual Review of Neuroscience, 34, 441-466.

Gitelman, D. R., Penny, W. D., Ashburner, J., \& Friston, K. J. (2003). Modeling regional and psychophysiologic interactions in fMRI: The importance of hemodynamic deconvolution. Neuroimage, 19, 200-207.

Glascher, J. (2009). Visualization of group inference data in functional neuroimaging. Neuroinformatics, 7, 73-82.

Glimcher, P. W. (2011). Understanding dopamine and reinforcement learning: The dopamine reward prediction error hypothesis. Proceedings of the National Academv of Sciences, U.S.A., 108(Suppl. 3), 15647-15654.

Goldstein, R. Z., Tomasi, D., Alia-Klein, N., Cottone, L. A., Zhang, L., Telang, F., et al. (2007). Subjective sensitivity to monetary gradients is associated with frontolimbic activation to reward in cocaine abusers. Drug and Alcohol Dependence, $87,233-240$

Gruber, A. J., Dayan, P., Gutkin, B. S., \& Solla, S. A. (2006). Dopamine modulation in the basal ganglia locks the gate to working memory. Iournal of Computational Neuroscience. 20, $153-166$

Haber, S. N. (2003). The primate basal ganglia: Parallel and integrative networks. Journal of Chemical Neuroanatomy 26, 317-330

Haber, S. N., Fudge, J. L., \& McFarland, N. R. (2000). Striatonigrostriatal pathways in primates form an ascending 
spiral from the shell to the dorsolateral striatum. Journal of Neuroscience, 20, 2369-2382.

Hazy, T. E., Frank, M. J., \& O’Reilly, R. C. (2007). Towards an executive without a homunculus: Computational models of the prefrontal cortex/basal ganglia system. Philosophical Transactions of the Royal Society of London, Series B, Biological Sciences, 362, 1601-1613.

Holmes, A. P., \& Friston, K. J. (1998). Generalisability, random effects and population inference. Neuroimage, 7, S754.

Howell, D. (2012). Statistical methods for psychology. Stanford, CT: Cengage Learning.

Jenkins, B. G. (2012). Pharmacologic magnetic resonance imaging (phMRI): Imaging drug action in the brain. Neuroimage, 62, 1072-1085.

Kennerley, S. W., \& Wallis, J. D. (2009a). Encoding of reward and space during a working memory task in the orbitofrontal cortex and anterior cingulate sulcus. Iournal of Neurophysiology. 102, 3352-3364.

Kennerley, S. W., \& Wallis, J. D. (2009b). Reward-dependent modulation of working memory in lateral prefrontal cortex. Lournal of Neuroscience, 29, 3259-3270.

Kleiner, M., Brainard, D., \& Pelli, D. (2007). What's new in Psychtoolbox-3? Perception, 36, 14.

Knutson, B., \& Gibbs, S. E. (2007). Linking nucleus accumbens dopamine and blood oxygenation. Psychopharmacology, 191, 813-822.

Krawczyk, D. C., \& D’Esposito, M. (2013). Modulation of working memory function by motivation through loss-aversion. Human Brain Mapping, 34, 762-774.

Krawczyk, D. C., Gazzaley, A., \& D'Esposito, M. (2007). Reward modulation of prefrontal and visual association cortex during an incentive working memory task. Brain Research, 1141, $168-177$.

Lawrence, A. D., Sahakian, B. J., \& Robbins, T. W. (1998). Cognitive functions and corticostriatal circuits: Insights from Huntington's disease. Trends in Cognitive Sciences, 2, 379-388.

Lund, T. E., Norgaard, M. D., Rostrup, E., Rowe, J. B., \& Paulson, O. B. (2005). Motion or activity: Their role in intra and inter-subject variation in fMRI. Neuroimage, 26, 960-964.

Maia, T. V., \& Frank, M. J. (2011). From reinforcement learning models to psychiatric and neurological disorders. Nature Neuroscience, 14, 154-162.

Marquand, A. F., De Simoni, S., O’Daly, O. G., Williams, S. C., Mourao-Miranda, J., \& Mehta, M. A. (2011). Pattern classification of working memory networks reveals differential effects of methylphenidate, atomoxetine, and placebo in healthy volunteers. Neuropsvchopharmacologv, 36, 1237-1247.

Moustafa, A. A., Sherman, S. J., \& Frank, M. J. (2008). A dopaminergic basis for working memory, learning and attentional shifting in Parkinsonism. Neuropsychologia. 46, 3144-3156.

Mumford, J. A., \& Nichols, T. (2009). Simple group fMRI modeling and inference. Neuroimage, 47, 1469-1475.

Murty, V. P., \& Adcock, R. A. (in press). Enriched encoding: Reward motivation organizes cortical networks for hippocampal detection of unexpected events. Cerebral Cortex.

Murty, V. P., Sambataro, F., Radulescu, E., Altamura, M., Iudicello, J., Zoltick, B., et al. (2011). Selective updating of working memory content modulates meso-cortico-striatal activity. Neuroimage, 57, 1264-1272.

Owen, A. M., Downes, J. J., Sahakian, B. J., Polkey, C. E., \& Robbins, T. W. (1990). Planning and spatial working memory following frontal lobe lesions in man. Neuropsvchologia. 28, 1021-1034.

Patenaude, B., Smith, S. M., Kennedy, D. N., \& Jenkinson, M. (2011). A Bayesian model of shape and appearance for subcortical brain segmentation. Neuroimage, 56, 907-922.

Petrides, M. (1996). Specialized systems for the processing of mnemonic information within the primate frontal cortex. Philosophical Transactions of the Royal Society of London, Series B, Biological Sciences, 351, 1455-1461; discussion 1461-1452.

Poser, B. A., Versluis, M. J., Hoogduin, J. M., \& Norris, D. G. (2006). BOLD contrast sensitivity enhancement and artifact reduction with multiecho EPI: Parallel-acquired inhomogeneity-desensitized fMRI. Magnetic Resonance in Medicine, 55, 1227-1235.

Robbins, T. W., \& Everitt, B. J. (1996). Neurobehavioural mechanisms of reward and motivation. Current Opinion in Neurobiology, 6, 228-236.

Roth, J. K., Serences, J. T., \& Courtney, S. M. (2006). Neural system for controlling the contents of object working memory in humans. Cerebral Cortex, 16, 1595-1603.

Rowe, J. B., Hughes, L., Ghosh, B. C., Eckstein, D., WilliamsGray, C. H., Fallon, S., et al. (2008). Parkinson's disease and dopaminergic therapy-Differential effects on movement, reward and cognition. Brain, 131, 2094-2105.

Schott, B. H., Minuzzi, L., Krebs, R. M., Elmenhorst, D., Lang, M., Winz, O. H., et al. (2008). Mesolimbic functional magnetic resonance imaging activations during reward anticipation correlate with reward-related ventral striatal dopamine release. Journal of Neuroscience, 28, 14311-14319.

Schultz, W., Dayan, P., \& Montague, P. R. (1997). A neural substrate of prediction and reward. Science, 275, 1593-1599.

Seamans, J. K., \& Yang, C. R. (2004). The principal features and mechanisms of dopamine modulation in the prefrontal cortex. Progress in Neurobiology, 74, 1-58.

Servan-Schreiber, D., Printz, H., \& Cohen, J. D. (1990). A network model of catecholamine effects: Gain, signal-tonoise ratio, and behavior. Science, 249, 892-895.

Tobler, P. N., Fiorillo, C. D., \& Schultz, W. (2005). Adaptive coding of reward value by dopamine neurons. Science, 307, 1642-1645

Tomasi, D., Goldstein, R. Z., Telang, F., Maloney, T., Alia-Klein, N., Caparelli, E. C., et al. (2007). Widespread disruption in brain activation patterns to a working memory task during cocaine abstinence. Brain Research, 1171, 83-92.

van Schouwenburg, M., Aarts, E., \& Cools, R. (2010). Dopaminergic modulation of cognitive control: Distinct roles for the prefrontal cortex and the basal ganglia. Current Pharmaceutical Design, 16, 2026-2032.

Volkow, N. D., Wang, G. J., Telang, F., Fowler, J. S., Logan, J., Jayne, M., et al. (2007). Profound decreases in dopamine release in striatum in detoxified alcoholics: Possible orbitofrontal involvement. Journal of Neuroscience, 27, 12700-12706.

Wise, R. A. (2004). Dopamine, learning and motivation. Nature Reviews Neuroscience, 5, 483-494.

Wittmann, B. C., Schott, B. H., Guderian, S., Frey, J. U., Heinze, H. J., \& Duzel, E. (2005). Reward-related fMRI activation of dopaminergic midbrain is associated with enhanced hippocampus-dependent long-term memory formation. Neuron, 45, 459-467. 


\section{This article has been cited by:}

1. Sean J. Fallon, Rozemarijn M. Mattiesing, Nina Dolfen, Sanjay G. Manohar, Masud Husain. 2018. Ignoring versus updating in working memory reveal differential roles of attention and feature binding. Cortex 107, 50-63. [Crossref]

2. Sean James Fallon, Rozemarijn Margaretha Mattiesing, Kinan Muhammed, Sanjay Manohar, Masud Husain. 2017. Fractionating the Neurocognitive Mechanisms Underlying Working Memory: Independent Effects of Dopamine and Parkinson's Disease. Cerebral Cortex 27:12, 5727-5738. [Crossref]

3. Rebecca A. Lundwall, James L. Dannemiller, H. Hill Goldsmith. 2017. Genetic associations with reflexive visual attention in infancy and childhood. Developmental Science 20:3, e12371. [Crossref]

4. Sean James Fallon, Nahid Zokaei, Agnes Norbury, Sanjay G. Manohar, Masud Husain. 2017. Dopamine Alters the Fidelity of Working Memory Representations according to Attentional Demands. Journal of Cognitive Neuroscience 29:4, 728-738. [Abstract] [Full Text] [PDF] [PDF Plus]

5. Sean James Fallon, Marieke E. van der Schaaf, Niels ter Huurne, Roshan Cools. 2017. The Neurocognitive Cost of Enhancing Cognition with Methylphenidate: Improved Distractor Resistance but Impaired Updating. Journal of Cognitive Neuroscience 29:4, 652-663. [Abstract] [Full Text] [PDF] [PDF Plus]

6. Elizabeth V. Goldfarb, Monja I. Froböse, Roshan Cools, Elizabeth A. Phelps. 2017. Stress and Cognitive Flexibility: Cortisol Increases Are Associated with Enhanced Updating but Impaired Switching. Journal of Cognitive Neuroscience 29:1, 14-24. [Abstract] [Full Text] [PDF] [PDF Plus]

7. Sean James Fallon, Adam Hampshire, Roger A. Barker, Adrian M. Owen. 2016. Learning to be inflexible: Enhanced attentional biases in Parkinson's disease. Cortex 82, 24-34. [Crossref]

8. Roshan Cools. 2016. The costs and benefits of brain dopamine for cognitive control. Wiley Interdisciplinary Reviews: Cognitive Science 7:5, 317-329. [Crossref]

9. Mitchell G. Uitvlugt, Timothy J. Pleskac, Susan M. Ravizza. 2016. The nature of working memory gating in Parkinson's disease: A multi-domain signal detection examination. Cognitive, Affective, \& Bebavioral Neuroscience 16:2, 289-301. [Crossref]

10. Sean James Fallon, Nahid Zokaei, Masud Husain. 2016. Causes and consequences of limitations in visual working memory. Annals of the New York Academy of Sciences 1369:1, 40-54. [Crossref]

11. Roshan Cools. 2015. The cost of dopamine for dynamic cognitive control. Current Opinion in Bebavioral Sciences 4, 152-159. [Crossref] 